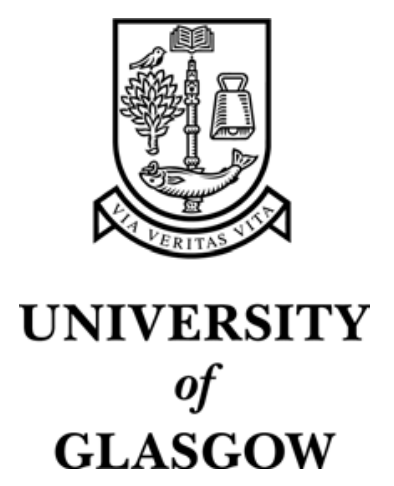

Abraham, David J and Irving, Robert W and Manlove, David F (2003)

The student-project allocation problem. In, Proceedings of ISAAC 2003:

the 14th Annual International Symposium on Algorithms and

Computation, 15-17 December, 2003 Lecture Notes in Computer Science Vol 2906, pages 474-484, Kyoto, Japan.

http://eprints.gla.ac.uk/archive/00001034/ 


\title{
The Student-Project Allocation Problem
}

\author{
David J. Abraham, Robert W. Irving, and David F. Manlove \\ Department of Computing Science, University of Glasgow, Glasgow G12 8QQ, UK \\ Email: \{dabraham,rwi,davidm\}@dcs.gla.ac.uk. Fax: +44 1413304913.
}

\begin{abstract}
We study the Student-Project Allocation problem (SPA), a generalisation of the classical Hospitals / Residents problem (HR). An instance of SPA involves a set of students, projects and lecturers. Each project is offered by a unique lecturer, and both projects and lecturers have capacity constraints. Students have preferences over projects, whilst lecturers have preferences over students. We present an optimal lineartime algorithm for allocating students to projects, subject to these preferences and capacities. In particular, the algorithm finds a stable matching of students to projects. Here, the concept of stability generalises the stability definition in the HR context. The stable matching produced by our algorithm is simultaneously best-possible for all students. The SPA problem model that we consider is very general and has applications to a range of different contexts besides student-project allocation.
\end{abstract}

\section{Introduction}

In many university departments, students seek a project in a given field of speciality as part of the upper level of their degree programme. Usually, a project can be filled by at most one student, though in some cases a project is suitable for more than one student to work on simultaneously. To give students something of a choice, there should be as wide a range of available projects as possible, and in any case the total number of project places should not be less than the total number of students. Typically a lecturer will also offer a range of projects, but does not necessarily expect that all will be taken up.

Each student has preferences over the available projects that he/she finds acceptable, whilst a lecturer will normally have preferences over the students that he/she is willing to supervise. There may also be upper bounds on the number of students that can be assigned to a particular project, and the number of students that a given lecturer is willing to supervise. In this paper we consider the problem of allocating students to projects based on these preference lists and capacity constraints - the so-called Student-Project Allocation problem (SPA).

SPA is an example of a two-sided matching problem [10], a large and very general class of problems in which the input set of participants can be partitioned into two disjoint sets $A$ and $B$ (in this case $A$ is the set of students and $B$ is the set of projects), and we seek to match members of $A$ to members of $B$, i.e. to find a subset of $A \times B$, subject to various criteria. These criteria usually involve

\footnotetext{
* Supported by award NUF-NAL-02 from the Nuffield Foundation, and grant
} GR/R84597/01 from the Engineering and Physical Sciences Research Council. 
capacity constraints, and/or preference lists, for example.

Both historical evidence (see e.g. [4, pp.3-4], [7]) and economic analysis [10] indicate that participants involved in two-sided matching problems should not be allowed to construct an allocation by approaching one another directly in order to make ad hoc arrangements. Instead, the allocation process should be automated by means of a centralised matching scheme. Moreover, it has been convincingly argued [9] that the key property that a matching constructed by such schemes should satisfy is stability. A formal definition of stability follows, but informally, a stable matching $M$ guarantees that no two participants who are not matched together in $M$ would rather be matched to one another than remain with their assignment in $M$. Such a pair of participants would form a private arrangement and would undermine the integrity of the matching.

The National Resident Matching Program (NRMP) [8] in the US is perhaps the largest and best-known example of a centralised matching scheme. It has been in operation since 1952, and currently handles the allocation of some 20,000 graduating medical students, or residents, to their first hospital posts, based on the preferences of residents over available hospital posts, and the preferences of hospital consultants over residents. The NRMP employs at its heart an efficient algorithm that essentially solves a variant of the classical Hospitals / Residents problem (HR) [3, 4]. The algorithm finds a stable matching of residents to hospitals that is resident-optimal, in that each resident obtains the best hospital that he/she could obtain in any stable matching.

There are many other examples of centralised matching schemes, both in educational and vocational contexts. Many university departments in particular seek to automate the allocation of students to projects. However, as we discuss in greater detail later, an optimal linear-time algorithm for this setting cannot be obtained by simply reducing an instance of SPA to an instance of HR. Thus, a specialised algorithm is required for the SPA problem.

In this paper we present a linear-time algorithm for finding a stable matching, given an instance of SPA. This algorithm is student-oriented, in that it finds a student-optimal stable matching. In this matching, each student obtains the best project that he/she could obtain in any stable matching. Our algorithm is applicable for any context that fits into the SPA model, for example where applicants seek posts at large organisations, each split into several departments.

The remainder of this paper is structured as follows. In Section 2, a formal definition of the SPA problem is given. Then, in Section 3, the algorithm for SPA is presented, together with correctness proofs and an analysis of its complexity. Finally, Section 4 contains some conclusions and open problems.

\section{Definition of the Student-Project Allocation Problem}

An instance of the Student-Project Allocation problem (SPA) may be defined as follows. Let $S=\left\{s_{1}, s_{2}, \ldots, s_{n}\right\}$ be a set of students, let $P=\left\{p_{1}, p_{2}, \ldots, p_{m}\right\}$ be a set of projects, and let $L=\left\{l_{1}, l_{2}, \ldots, l_{q}\right\}$ be a set of lecturers. Each student $s_{i}$ supplies a preference list, ranking a subset of $P$ in strict order. If project $p_{j}$ appears on $s_{i}$ 's preference list, we say that $s_{i}$ finds $p_{j}$ acceptable. Denote by $A_{i}$ 


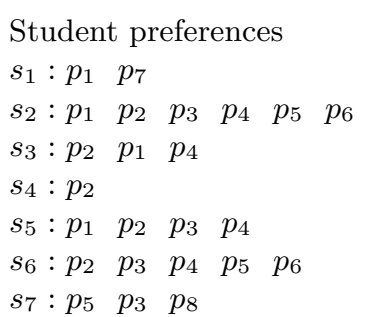

Lecturer preferences

$l_{1}: s_{7} \quad s_{4} \quad s_{1} \quad s_{3} \quad s_{2} \quad s_{5} \quad s_{6} \quad l_{1}$ offers $p_{1}, p_{2}, p_{3}$

$l_{2}: s_{3} s_{2} \quad s_{6} \quad s_{7} s_{5}$

$l_{3}: s_{1} s_{7}$

$l_{2}$ offers $p_{4}, p_{5}, p_{6}$

$l_{3}$ offers $p_{7}, p_{8}$

Project capacities: $c_{1}=2, c_{i}=1(2 \leq i \leq 8)$

Lecturer capacities: $d_{1}=3, d_{2}=2, \bar{d}_{3}=2$

Fig. 1. An instance of the Student-Project Allocation problem.

the set of projects that $s_{i}$ finds acceptable.

Each lecturer $l_{k}$ offers a non-empty set of projects $P_{k}$, where $P_{1}, P_{2}, \ldots, P_{q}$ partitions $P$. Let $B_{k}=\left\{s_{i} \in S: P_{k} \cap A_{i} \neq \emptyset\right\}$ (i.e. $B_{k}$ is the set of students who find acceptable a project offered by $l_{k}$ ). Lecturer $l_{k}$ supplies a preference list, denoted by $\mathcal{L}_{k}$, ranking $B_{k}$ in strict order. For any $p_{j} \in P_{k}$, we denote by $\mathcal{L}_{k}^{j}$ the projected preference list of $l_{k}$ for $p_{j}$ - this is obtained from $\mathcal{L}_{k}$ by deleting those students who do not find $p_{j}$ acceptable. In this way, the ranking of $\mathcal{L}_{k}^{j}$ is inherited from $\mathcal{L}_{k}$. Also, $l_{k}$ has a capacity constraint $d_{k}$, indicating the maximum number of students that he/she is willing to supervise. Similarly, each project $p_{j}$ carries a capacity constraint $c_{j}$, indicating the maximum number of students that could be assigned to $p_{j}$. We assume that $\max \left\{c_{j}: p_{j} \in P_{k}\right\} \leq d_{k}$.

An example SPA instance is shown in Figure 1. Here the set of students is $S=$ $\left\{s_{1}, s_{2}, \ldots, s_{7}\right\}$, the set of projects is $P=\left\{p_{1}, p_{2}, \ldots, p_{8}\right\}$ and the set of lecturers is $L=\left\{l_{1}, l_{2}, l_{3}\right\}$. Lecturers offer projects as indicated, and the preference lists and capacity constaints are also shown. As an example, the projected preference list of $l_{1}$ for $p_{1}$ comprises $s_{1}, s_{3}, s_{2}, s_{5}$, ranked in that order.

An assignment $M$ is a subset of $S \times P$ such that:

1. $\left(s_{i}, p_{j}\right) \in M$ implies that $p_{j} \in A_{i}$ (i.e. $s_{i}$ finds $p_{j}$ acceptable).

2. For each student $s_{i} \in S,\left|\left\{\left(s_{i}, p_{j}\right) \in M: p_{j} \in P\right\}\right| \leq 1$.

If $\left(s_{i}, p_{j}\right) \in M$, we say that $s_{i}$ is assigned to $p_{j}$, and $p_{j}$ is assigned $s_{i}$. Hence Condition 2 states that each student is assigned to at most one project in $M$. For notational convenience, if $s_{i}$ is assigned in $M$ to $p_{j}$, we may also say that $s_{i}$ is assigned to $l_{k}$, and $l_{k}$ is assigned $s_{i}$, where $p_{j} \in P_{k}$.

For any student $s_{i} \in S$, if $s_{i}$ is assigned in $M$ to some project $p_{j}$, we let $M\left(s_{i}\right)$ denote $p_{j}$; otherwise we say that $s_{i}$ is unmatched in $M$. For any project $p_{j} \in P$, we denote by $M\left(p_{j}\right)$ the set of students assigned to $p_{j}$ in $M$. Project $p_{j}$ is under-subscribed, full or over-subscribed according as $\left|M\left(p_{j}\right)\right|$ is less than, equal to, or greater than $c_{j}$, respectively. Similarly, for any lecturer $l_{k} \in L$, we denote by $M\left(l_{k}\right)$ the set of students assigned to $l_{k}$ in $M$. Lecturer $l_{k}$ is undersubscribed, full or over-subscribed according as $\left|M\left(l_{k}\right)\right|$ is less than, equal to, or greater than $d_{k}$ respectively.

A matching $M$ is an assignment such that:

3. For each project $p_{j} \in P,\left|\left\{\left(s_{i}, p_{j}\right) \in M: s_{i} \in S\right\}\right| \leq c_{j}$.

4. For each lecturer $l_{k} \in L,\left|\left\{\left(s_{i}, p_{j}\right) \in M: s_{i} \in S \wedge \bar{p}_{j} \in P_{k}\right\}\right| \leq d_{k}$. 
Hence Condition 3 stipulates that $p_{j}$ is assigned at most $c_{j}$ students in $M$, whilst Condition 4 requires that $l_{k}$ is assigned at most $d_{k}$ students in $M$.

A blocking pair relative to a matching $M$ is a (student,project) pair $\left(s_{i}, p_{j}\right) \in$ $(S \times P) \backslash M$ such that:

1. $p_{j} \in A_{i}$ (i.e. $s_{i}$ finds $p_{j}$ acceptable).

2. Either $s_{i}$ is unmatched in $M$, or $s_{i}$ prefers $p_{j}$ to $M\left(s_{i}\right)$.

3. Either

(a) $p_{j}$ is under-subscribed and $l_{k}$ is under-subscribed, or

(b) $p_{j}$ is under-subscribed, $l_{k}$ is full, and either $l_{k}$ prefers $s_{i}$ to the worst student $s^{\prime}$ in $M\left(l_{k}\right)$ or $s_{i}=s^{\prime}$, or

(c) $p_{j}$ is full and $l_{k}$ prefers $s_{i}$ to the worst student in $M\left(p_{j}\right)$,

where $l_{k}$ is the lecturer who offers $p_{j}$.

A matching is stable if it admits no blocking pair. We now give some intuition for the definition of a blocking pair. Suppose that $\left(s_{i}, p_{j}\right)$ forms a blocking pair with respect to matching $M$, and let $l_{k}$ be the lecturer who offers $p_{j}$.

In general we assume that $s_{i}$ prefers to be matched to an acceptable project rather than to remain unmatched. Hence Condition 2 indicates the means by which a student could improve relative to $M$. Suppose now that this condition is satisfied. To explain Condition 3(a), matching $M$ cannot be stable if each of project $p_{j}$ and lecturer $l_{k}$ has a free place to take on $s_{i}$ (or to let $s_{i}$ change projects offered by $l_{k}$ ). We now consider Condition $3(\mathrm{~b})$. If $p_{j}$ is under-subscribed, $l_{k}$ is full, and $s_{i}$ was not already matched in $M$ to a project offered by $l_{k}$, then $l_{k}$ cannot take on $s_{i}$ without first rejecting at least one student. Lecturer $l_{k}$ would only agree to this switch if he/she prefers $s_{i}$ to the worst student assigned to $l_{k}$ in $M$. In this case, project $p_{j}$ has room for $s_{i}$. Alternatively, if $s_{i}$ was already matched in $M$ to a project offered by $l_{k}$, then the total number of students assigned to $l_{k}$ remains the same, and $l_{k}$ agrees to the switch since $p_{j}$ has room for $s_{i}$. Finally, we consider Condition $3(\mathrm{c})$. If $p_{j}$ is full, then $l_{k}$ cannot take on $s_{i}$ without first rejecting at least one student assigned to $p_{j}$. Lecturer $l_{k}$ would only agree to this switch if he/she prefers $s_{i}$ to the worst student assigned to $p_{j}$ in $M$. Notice that if $s_{i}$ was already matched in $M$ to a project offered by $l_{k}$, then the number of students assigned to $l_{k}$ would decrease by 1 after the switch. However we argue that this is the "correct" definition of a blocking pair in this case, also having the side-effects of avoiding issues of strategy and maintaining useful structural properties. For a full discussion of this point, we refer the reader to Section 4.1 of [1].

We remark that HR is a special case of SPA in which $m=q, c_{j}=d_{j}$ and $P_{j}=\left\{p_{j}\right\}(1 \leq j \leq m)$. Essentially the projects and lecturers are indistinguishable in this case. In the HR setting, lecturers / projects are referred to as hospitals, and students are referred to as residents. Linear-time algorithms are known for finding a stable matching, given an instance of HR. The residentoriented algorithm [4, Section 1.6.3] finds a resident-optimal stable matching. In this stable matching, each matched resident is assigned to the best hospital that he/she could obtain in any stable matching, whilst each unmatched resident is unmatched in every stable matching. On the other hand, the hospital-oriented 
algorithm [4, Section 1.6.2] finds a hospital-optimal stable matching. In this stable matching, each full hospital is assigned the best set of residents that it could obtain in any stable matching, whilst each under-subscribed hospital is assigned the same set of residents in every stable matching.

It is worth drawing attention to a special case of HR (and hence of SPA). This is the classical Stable Marriage problem with Incomplete lists (SMI), where $c_{j}=1(1 \leq j \leq m)[3],[4$, Section 1.4.2]. In this setting, residents are referred to as men and hospitals are referred to as women. There exists a reduction from HR to SMI using the method of 'cloning' hospitals. That is, replace each hospital $h_{j}$, of capacity $c_{j}$, with $c_{j}$ women, denoted by $h_{j}^{1}, h_{j}^{2}, \ldots, h_{j}^{c_{j}}$. The preference list of $h_{j}^{k}$ is identical to the preference list of $h_{j}$. Any occurrence of $h_{j}$ in a resident's preference list should be replaced by $h_{j}^{1}, h_{j}^{2}, \ldots, h_{j}^{c_{j}}$ in that order. Hence in theory, the Gale / Shapley algorithm for SMI [4, Section 1.4.2] could be used to solve an HR instance. However in practice direct algorithms are applied to HR instances [4, Section 1.6], because the cloning technique increases the number of hospitals (women) in a given HR instance by a potentially significant factor of $C / m$, where $C=\sum_{j=1}^{j=m} c_{j}$.

On the other hand there is no straightforward reduction involving cloning from an instance of SPA to an instance of HR, due to the projects and lecturers being distinct entities, each having capacity constraints. Even if such a reduction were possible, again it would typically increase the number of lecturers (hospitals) by a significant factor. This justifies the approach of this paper, in which we consider a direct algorithm for SPA.

The running time of our algorithm is $O(L)$, where $L$ is the total length of the input preference lists, and hence is linear in the size of the problem instance. This algorithm is optimal, since the Stable Marriage problem (SM) - the special case of SMI in which $m=n$ and each student finds every project acceptable is a special case of SPA. A lower bound of $\Omega(L)$ is known for SM [6], and hence this also applies to SPA.

\section{The algorithm for SPA}

\subsection{Overview}

The algorithm for finding a student-optimal stable matching involves a sequence of apply operations (i.e. students apply to projects). An apply operation is similar to a proposal in the context of the Gale / Shapley algorithm for SM [3]. These operations lead to provisional assignments between students, projects and lecturers; such assignments can subsequently be broken during the algorithm's execution. Also, throughout the execution, entries are possibly deleted from the preference lists of students, and from the projected preference lists of lecturers. We use the abbreviation delete the pair $\left(s_{i}, p_{j}\right)$ to denote the operation of deleting $p_{j}$ from the preference list of $s_{i}$, and deleting $s_{i}$ from $\mathcal{L}_{k}^{j}$, where $l_{k}$ is the lecturer who offers $p_{j}$.

Initially all students are free, and all projects and lecturers are totally unsubscribed. As long as there is some student $s_{i}$ who is free and who has a non-empty 


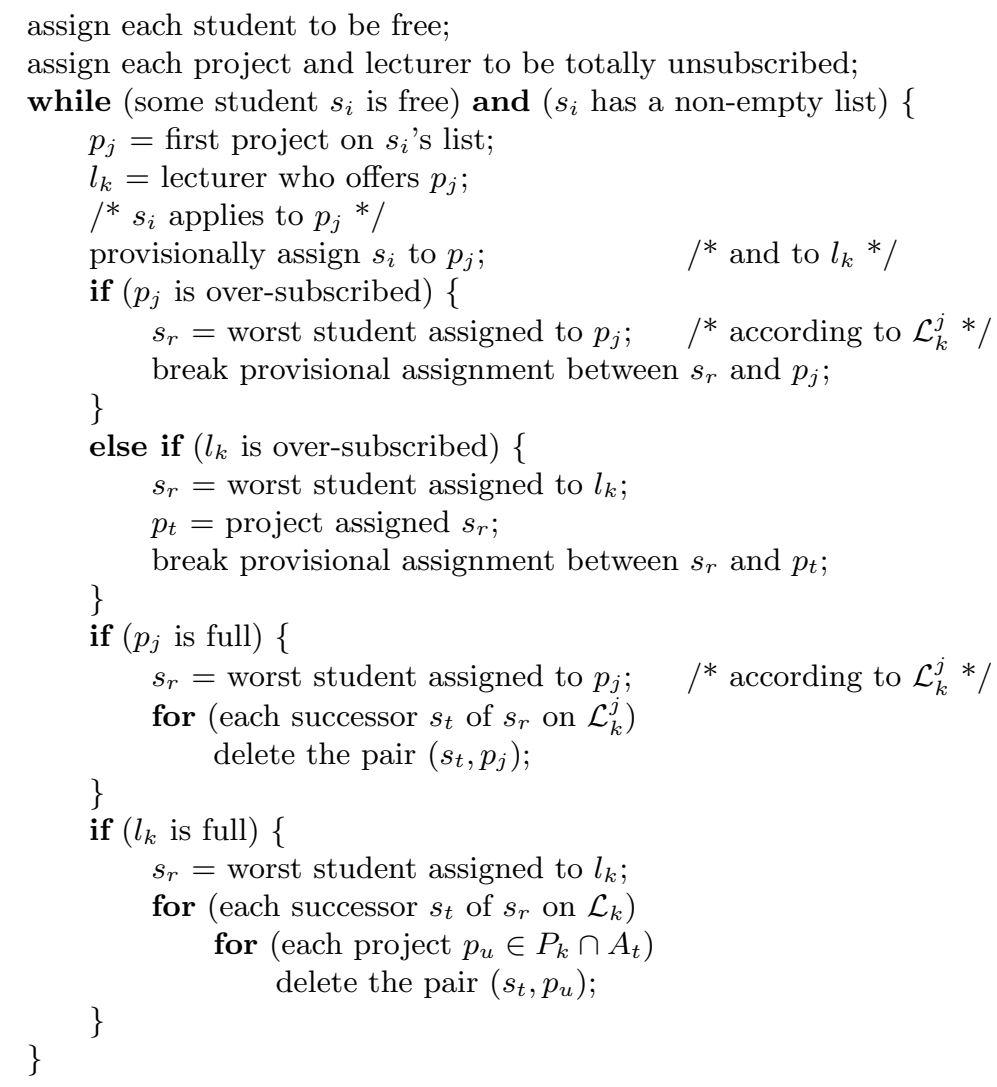

Fig. 2. Algorithm SPA-student for finding a student-optimal stable matching

list, $s_{i}$ applies to the first project $p_{j}$ on his/her list. We let $l_{k}$ be the lecturer who offers $p_{j}$. Immediately, $s_{i}$ becomes provisionally assigned to $p_{j}$ (and to $l_{k}$ ).

If $p_{j}$ is over-subscribed, then $l_{k}$ rejects the worst student $s_{r}$ assigned to $p_{j}$. The pair $\left(s_{r}, p_{j}\right)$ will be deleted by the subsequent conditional that tests for $p_{j}$ being full. Similarly, if $l_{k}$ is over-subscribed, then $l_{k}$ rejects his/her worst assigned student $s_{r}$. The pair $\left(s_{r}, p_{t}\right)$ will be deleted by either of the two subsequent conditionals, where $p_{t}$ was the project formerly assigned to $s_{r}$.

Regardless of whether any rejections occurred as a result of the two situations described in the previous paragraph, we have two further (possibly non-disjoint) cases in which deletions may occur. If $p_{j}$ is full, we let $s_{r}$ be the worst student assigned to $p_{j}$ (according to $\mathcal{L}_{k}^{j}$ ) and delete the pair $\left(s_{t}, p_{j}\right)$ for each successor $s_{t}$ of $s_{r}$ on $\mathcal{L}_{k}^{j}$. Similarly if $l_{k}$ is full, we let $s_{r}$ be the worst student assigned to $l_{k}$, and delete the pair $\left(s_{t}, p_{u}\right)$ for each successor $s_{t}$ of $s_{r}$ on $\mathcal{L}_{k}$, and for each project $p_{u}$ offered by $l_{k}$ that $s_{t}$ finds acceptable.

The algorithm is described in pseudocode form in Figure 2 as Algorithm SPA-student. We will prove that, once the main loop terminates, the assigned pairs constitute a student-optimal stable matching. 


\subsection{Correctness of Algorithm SPA-student}

We firstly remark that Algorithm SPA-student terminates with a matching. The correctness of the algorithm, together with the optimality property of the constructed matching, may be established by the following sequence of lemmas.

Lemma 1. No pair deleted during an execution of Algorithm SPA-student can block the constructed matching.

Proof. Let $E$ be an arbitrary execution of the algorithm in which some pair $\left(s_{i}, p_{j}\right)$ is deleted. Suppose for a contradiction that $\left(s_{i}, p_{j}\right)$ blocks $M$, the matching generated by $E$. Now, $\left(s_{i}, p_{j}\right)$ is deleted in $E$ because either (i) $p_{j}$ becomes full, or (ii) $l_{k}$ becomes full, where $l_{k}$ is the lecturer offering $p_{j}$. In Case (i), it turns out that $\left(s_{i}, p_{j}\right)$ fails (a), (b) and (c) of Condition 3 of a blocking pair, a contradiction. The details for each of these sub-cases are omitted here for space reasons, but may be found in [1]. Case (ii) is easier: $\left(s_{i}, p_{j}\right)$ cannot block $M$, since once full, a lecturer never becomes under-subscribed, and is only ever assigned more preferable students.

Lemma 2. Algorithm SPA-student generates a stable matching.

Proof. Let $M$ be the matching generated by an arbitrary execution $E$ of the algorithm, and let $\left(s_{i}, p_{j}\right)$ be any pair blocking $M$. We will show that $\left(s_{i}, p_{j}\right)$ must be deleted in $E$, thereby contradicting Lemma 1. For, suppose not. Then $s_{i}$ must be matched to some project $M\left(s_{i}\right) \neq p_{j}$, for otherwise $s_{i}$ is free with a non-empty preference list (containing $p_{j}$ ), thereby contradicting the fact that the algorithm terminates. Now, when $s_{i}$ applies to $M\left(s_{i}\right), M\left(s_{i}\right)$ is the first project on his/her list. Hence, $\left(s_{i}, p_{j}\right)$ must be deleted, since for $\left(s_{i}, p_{j}\right)$ to block $M, s_{i}$ must prefer $p_{j}$ to $M\left(s_{i}\right)$.

Lemma 3. No stable pair (i.e. (student,project) pair belonging to some stable matching) is deleted during an execution of Algorithm SPA-student.

Proof. Suppose for a contradiction that $\left(s_{i}, p_{j}\right)$ is the first stable pair deleted during an arbitrary execution $E$ of the algorithm. Let $M$ be the matching immediately after the deletion in $E$, and let $M^{\prime}$ be any stable matching containing $\left(s_{i}, p_{j}\right)$. Now, $\left(s_{i}, p_{j}\right)$ is deleted in $E$ because either (i) $p_{j}$ becomes full, or (ii) $l_{k}$ becomes full, where $l_{k}$ is the lecturer offering $p_{j}$. We consider each case in turn.

(i) Suppose $\left(s_{i}, p_{j}\right)$ is deleted because $p_{j}$ becomes full during $E$. Immediately after the deletion, $p_{j}$ is full, and $l_{k}$ prefers all students in $M\left(p_{j}\right)$ to $s_{i}$. Now, $s_{i} \in M^{\prime}\left(p_{j}\right) \backslash M\left(p_{j}\right)$, and since $p_{j}$ is full in $M$, there must be some $s \in$ $M\left(p_{j}\right) \backslash M^{\prime}\left(p_{j}\right)$. We will show that $\left(s, p_{j}\right)$ forms a blocking pair, contradicting the stability of $M^{\prime}$.

Firstly, since $\left(s_{i}, p_{j}\right)$ is the first stable pair deleted in $E, s$ prefers $p_{j}$ to any of his/her stable partners (except possibly for $p_{j}$ itself). Additionally, since $\left(s_{i}, p_{j}\right) \in M^{\prime}$ and $l_{k}$ prefers $s$ to $s_{i}$, it follows that $l_{k}$ prefers $s$ to both the worst student in $M^{\prime}\left(p_{j}\right)$ and $M^{\prime}\left(l_{k}\right)$. Clearly then, for any combination of $l_{k}$ and $p_{j}$ being full or under-subscribed, $\left(s, p_{j}\right)$ satisfies all the conditions to block $M^{\prime}$. 
(ii) Suppose that $\left(s_{i}, p_{j}\right)$ is deleted because $l_{k}$ becomes full during $E$. Immediately after the deletion, $l_{k}$ is full, and $l_{k}$ prefers all students in $M\left(l_{k}\right)$ to $s_{i}$. We consider two cases: $\left|M^{\prime}\left(p_{j}\right)\right|>\left|M\left(p_{j}\right)\right|$ and $\left|M^{\prime}\left(p_{j}\right)\right| \leq\left|M\left(p_{j}\right)\right|$.

Suppose firstly $\left|M^{\prime}\left(p_{j}\right)\right|>\left|M\left(p_{j}\right)\right|$. Since $l_{k}$ is full in $M$, and $\left(s_{i}, p_{j}\right) \notin M$, there must be some project $p \in P_{k} \backslash\left\{p_{j}\right\}$ such that $\left|M^{\prime}(p)\right|<|M(p)|$. We remark that $p$ is therefore under-subscribed in $M^{\prime}$. Now, let $s$ be any student in $M(p) \backslash M^{\prime}(p)$. Since $\left(s_{i}, p_{j}\right)$ is the first stable pair deleted, $s$ prefers $p$ to any of his/her stable partners (except possibly for $p$ itself). Also, $l_{k}$ prefers $s$ to $s_{i}$, and hence to the worst student in $M^{\prime}\left(l_{k}\right)$. So, in either case that $l_{k}$ is full or under-subscribed, $(s, p)$ blocks $M^{\prime}$.

Now suppose $\left|M^{\prime}\left(p_{j}\right)\right| \leq\left|M\left(p_{j}\right)\right|$. Then there is some $s \neq s_{i} \in M\left(p_{j}\right) \backslash M^{\prime}\left(p_{j}\right)$. Now, $p_{j}$ is under-subscribed in $M$, for otherwise $\left(s_{i}, p_{j}\right)$ is deleted because $p_{j}$ becomes full, contradicting the assumption that deletion occurs because $l_{k}$ becomes full. Therefore, $p_{j}$ is under-subscribed in $M^{\prime}$. As above, $s$ prefers $p_{j}$ to any of his/her stable partners (except possibly for $p_{j}$ itself), since $\left(s_{i}, p_{j}\right)$ is the first stable pair deleted. Also, $l_{k}$ prefers $s$ to $s_{i}$, and hence to the worst pair in $M^{\prime}\left(l_{k}\right)$. So, in either case that $l_{k}$ is full or under-subscribed, $\left(s, p_{j}\right)$ blocks $M^{\prime}$.

The following theorem collects together Lemmas 1-3.

Theorem 1. For a given instance of SPA, any execution of Algorithm SPAstudent constructs the student-optimal stable matching.

Proof. Let $M$ be a matching generated by an arbitrary execution $E$ of the algorithm. In $M$, each student is assigned to the first project on his/her reduced preference list, if any. By Lemma 2, $M$ is stable, and so each of these (student, project) pairs is stable. Also, by Lemma 3, no stable pair is deleted during $E$. It follows then that in $M$, each student is assigned to the best project that he/she can obtain in any stable matching.

For example, in the SPA instance given by Figure 1, the student-optimal stable matching is $\left\{\left(s_{1}, p_{1}\right),\left(s_{2}, p_{5}\right),\left(s_{3}, p_{4}\right),\left(s_{4}, p_{2}\right),\left(s_{7}, p_{3}\right)\right\}$.

We now state a result similar to the 'Rural Hospitals Theorem' for HR [4, Theorem 1.6.3]. In particular, the following theorem indicates that, in no other stable matching could we match a different set of students than that matched by Algorithm SPA-student. The proof is omitted here for space reasons, but may be found in [1].

Theorem 2. For a given SPA instance:

(i) each lecturer has the same number of students in all stable matchings;

(ii) exactly the same students are unmatched in all stable matchings;

(iii) a project offered by an under-subscribed lecturer has the same number of students in all stable matchings.

However it turns out that an under-subscribed lecturer need not obtain the same set of students in all stable matchings, and in addition, a project offered by a full lecturer need not obtain the same number of students in all stable matchings. Example SPA instances illustrating these remarks are given in [1]. 


\subsection{Analysis of Algorithm SPA-student}

The algorithm's time complexity depends on how efficiently we can execute 'apply' operations and deletions, each of which occur at most once for any (student, project) pair. It turns out that both operations can be implemented to run in constant time, giving an overall time complexity of $\Theta(L)$, where $L$ is the total length of all the preference lists. We briefly outline the non-trivial aspects of such an implementation.

For each student $s_{i}$, build an array, $\operatorname{rank}_{s_{i}}$, where $\operatorname{rank}_{s_{i}}\left(p_{j}\right)$ is the index of project $p_{j}$ in $s_{i}$ 's preference list. Represent $s_{i}$ 's preference list by embedding doubly linked lists in an array, preference $_{s_{i}}$. For each project $p_{j} \in A_{i}$, preference $_{s_{i}}\left(\operatorname{rank}_{s_{i}}\left(p_{j}\right)\right)$ stores the list node containing $p_{j}$. This node contains two next pointers (and two previous pointers) - one to the next project in $s_{i}$ 's list (after deletions, this project may not located at the next array position), and another pointer to the next project $p^{\prime}$ in $s_{i}$ 's list, where $p^{\prime}$ and $p_{j}$ are both offered by the same lecturer. Construct this list by traversing through $s_{i}$ 's preference list, using a temporary array to record the last project in the list offered by each lecturer. Use virtual initialisation (described in [2, p.149]) for these arrays, since the overall $\Theta(n q)$ initialisation cost may be super-linear in $L$. Clearly, using these data structures, we can find and delete a project from a given student in constant time, as well as efficiently delete all projects offered by a given lecturer.

Represent each lecturer $l_{k}$ 's preference list $\mathcal{L}_{k}$ by an array preference $_{l_{k}}$, with an additional pointer, last $_{l_{k}}$. Initially, last $_{l_{k}}$ stores the index of the last position in preference $_{l_{k}}$. However, once $l_{k}$ is full, make last $_{l_{k}}$ equivalent to $l_{k}$ 's worst assigned student through the following method. Perform a backwards linear traversal through preference $l_{k}$, starting at last $_{l_{k}}$, and continuing until $l_{k}$ 's worst assigned student is encountered (each student stores a pointer to their assigned project, or a special null value if unassigned). All but the last student on this traversal must be deleted, and so the cost of the traversal may be attributed to the cost of the deletions in the student preference lists.

For each project $p_{j}$ offered by $l_{k}$, construct a preference array corresponding to $\mathcal{L}_{k}^{j}$. These project preference arrays are used in much the same way as the lecturer preference array, with one exception. When a lecturer $l_{k}$ becomes over-subscribed, the algorithm frees $l_{k}$ 's worst assigned student $s_{i}$ and breaks the assignment of $s_{i}$ to some project $p_{j}$. If $p_{j}$ was full, then it is now undersubscribed, and last $_{p_{j}}$ is no longer equivalent to $p_{j}$ 's worst assigned student. Rather than update last $_{p_{j}}$ immediately, which could be expensive, wait until $p_{j}$ is full again. The update then involves the same backwards linear traversal described above for $l_{k}$, although we must be careful not to delete pairs already deleted in one of $l_{k}$ 's traversals. Since we only visit a student at most twice during these backwards traversals, once for the lecturer and once for the project, the asymptotic running time remains linear.

The implementation issues discussed above lead to the following conclusion.

Theorem 3. Algorithm SPA-student may be implemented to use $\Theta(L)$ time and space, where $L$ is the total length of the preference lists in a given SPA instance. 


\section{Conclusions and open problems}

In this paper we have presented a student-oriented algorithm for a SPA instance. This produces the student-optimal stable matching, in which each student obtains the best project that he/she could obtain in any stable matching. We remark that we have also formulated a lecturer-oriented counterpart, which we omit for space reasons. This second algorithm produces the lecturer-optimal stable matching, in which each lecturer obtains the best set of students that he/she could obtain in any stable matching.

A number of interesting open problems remain. These include:

- The SPA model may be extended to the case where lecturers have preferences over (student,project) pairs. However in this setting it is an open problem to formulate an acceptable stability definition that avoids issues of strategy. For example, a student could deliberately shorten his/her preference list in order to obtain a better project, rather than submitting his/her true preferences. These strategic issues are described in more detail in [1].

- If we allow ties in the preference lists of students and lecturers, different stability definitions are possible. These can be obtained by extending stability definitions that have been applied to the Hospitals / Residents problem with Ties [5]. It remains open to construct algorithms for SPA where preference lists contain ties, under each of these stability criteria.

\section{References}

1. D.J. Abraham, R.W. Irving, and D.F. Manlove. The Student-Project Allocation Problem. Technical Report TR-2003-141 of the Computing Science Department of Glasgow University, 2003.

2. G. Brassard and P. Bratley. Fundamentals of Algorithmics. Prentice-Hall, 1996.

3. D. Gale and L.S. Shapley. College admissions and the stability of marriage. American Mathematical Monthly, 69:9-15, 1962.

4. D. Gusfield and R.W. Irving. The Stable Marriage Problem: Structure and Algorithms. MIT Press, 1989.

5. R.W. Irving, D.F. Manlove, and S. Scott. Strong stability in the Hospitals/Residents problem. In Proceedings of STACS 2003: the 20th International Symposium on Theoretical Aspects of Computer Science, volume 2607 of Lecture Notes in Computer Science, pages 439-450. Springer-Verlag, 2003.

6. C. Ng and D.S. Hirschberg. Lower bounds for the stable marriage problem and its variants. SIAM Journal on Computing, 19:71-77, 1990.

7. National Resident Matching Program. About the NRMP. Web document available at http://www.nrmp.org/about_nrmp/how.html.

8. National Resident Matching Program. Why the Match? Web document available at http://www.nrmp.org/whythematch.pdf.

9. A.E. Roth. The evolution of the labor market for medical interns and residents: a case study in game theory. Journal of Political Economy, 92(6):991-1016, 1984.

10. A.E. Roth and M.A.O. Sotomayor. Two-sided matching: a study in game-theoretic modeling and analysis, volume 18 of Econometric Society Monographs. Cambridge University Press, 1990. 